\title{
Complex Fresnel hologram display using a single SLM
}

\author{
Jung-Ping Liu, ${ }^{1, *}$ Wang-Yu Hsieh, ${ }^{1}$ Ting-Chung Poon, ${ }^{1,2}$ and Peter Tsang ${ }^{3}$ \\ 'Department of Photonics, Feng Chia University, No. 100 Wenhwa Rd., Taichung 407, Taiwan \\ ${ }^{2}$ Bradley Department of Electrical and Computer Engineering, Virginia Tech, Blacksburg, Virginia 24061, USA \\ ${ }^{3}$ Department of Electronic Engineering, City University of Hong Kong, Hong Kong, China \\ ${ }^{*}$ Corresponding author: jpliu@ @fu.edu.tw
}

Received 1 August 2011; revised 5 October 2011; accepted 18 October 2011; posted 21 October 2011 (Doc. ID 152128); published 17 November 2011

\begin{abstract}
We propose a novel optical method to display a complex Fresnel hologram using a single spatial light modulator (SLM). The method consists of a standard coherent image processing system with a sinusoidal grating at the Fourier plane. Two or three position-shifted amplitude holograms displayed at the input plane of the processing system can be coupled via the grating and will be precisely overlapped at the system's output plane. As a result, we can synthesize a complex hologram that is free of the twin image and the zero-order light using a single SLM. Because the twin image is not removed via filtering, the full bandwidth of the SLM can be utilized for displaying on-axis holograms. In addition, the degree of freedom of the synthesized complex hologram display can be extended by involving more than three amplitude holograms. (C) 2011 Optical Society of America
\end{abstract}

OCIS codes: $\quad 090.1760,090.1995,090.2870,120.2040$.

\section{Introduction}

In holography, the phase as well as the amplitude of an object light is encoded in the interference fringes as holographic gratings [1]. The object light can be retrieved by illuminating the hologram with the reference light involved in the recording process. Because a hologram contains the complete information of the object light, it is able to show a real three-dimensional scene, in contrast to photography or multiview parallax techniques. However, the amplitude-type on-axis hologram contains not only the desired object light but also the annoying zero-order light and the twin image. In conventional holography, thanks to the offaxis recording scheme [2], the object light, the zeroorder light, and the twin image can be separated spatially (or in the viewing angle) so that the reconstructed three-dimensional scene can be observed with little disturbance.

Although static holograms with high fidelity have been demonstrated for a long time, the display of high-quality digital holograms is a challenge. The

0003-6935/11/34H128-08\$15.00/0

(C) 2011 Optical Society of America display of digital holograms demands an array electro-optic device, such as a spatial light modulator (SLM). An ideal SLM should have a large clear aperture with uniform optical properties; the pixel pitch should be as small as possible to ensure a large bandwidth for displaying an off-axis hologram. A liquid crystal (LC) SLM is the most general type of SLM for holographic display. However, the size and pixel pitch of the commercially available LC SLMs are about $1 \mathrm{~cm} \times 2 \mathrm{~cm}$ and $10 \mu \mathrm{m}$, respectively, which limit the field of view, the bandwidth of the hologram, and the display quality.

There are some ways to overcome the limitations of SLMs, such as using a single SLM in conjunction with a scanning module or using multiple SLMs [3-7]. Suitable optical filtering is also able to achieve a single-sideband hologram [8,9]. But the limitation of the bandwidth is the same as that in displaying an off-axis hologram. Another way to remove the zeroorder light and the twin image is to create a complex hologram. The reconstruction of the complex hologram is free of the zero-order light and the twin image [10]. Unfortunately, current SLMs can modulate either phase or intensity of an incident light, but not both $[11,12]$. Some methods are proposed to alleviate 
the practical limitation in modulation [13-21]. But, in these methods, the holograms need to be recalculated to match the specific display systems. Using two SLMs can display a complex hologram directly. This can be implemented by cascading two SLMs, one for amplitude modulation (AM) and the other for phase modulation (PM) [22-24]. Alternatively, a beam splitter can be applied to combine the beams from two SLMs, one for displaying the real part and the other the imaginary part of the complex hologram [25-27].

In this paper, we propose a method to display a complex hologram using a single SLM. The method involves a standard coherent image processing system with a grating at the Fourier plane. Two positionshifted amplitude holograms displayed on a single SLM can be coupled via the grating and automatically overlapped at the output plane of the system to synthesize a complex hologram. The paper is organized as follows. In Section 2 , we introduce the concept of complex hologram and the proposed system. We then investigate that the system can combine not only two holograms but also multiple holograms. In Section 3, we carry out a series of simulations to verify our proposed method. The tolerance analysis and experimental demonstration of the synthesis of a complex hologram are provided in Section 4. Finally, we make some concluding remarks in Section $\underline{5}$.

\section{Display of Complex Fresnel Holograms: Principle}

\section{A. Complex Fresnel Hologram}

A conventional amplitude hologram is a collection of gratings with amplitude transmittance proportional to the interference fringes formed by the object light and the reference light. Mathematically, the amplitude hologram can be expressed as

$$
H=|O+R|^{2}=|O|^{2}+|R|^{2}+O R^{*}+O^{*} R,
$$

where $O$ and $R$ are the complex amplitudes of the object light and the reference light, respectively, and the asterisk stands for complex conjugate. In Eq. (1), the first two terms are the zero-order light, and the third and fourth terms are the stored original object light and the twin image, respectively. Thus, there is always the zero-order light and the twin image upon holographic reconstruction. If we can somehow remove the zero-order light and the twin image, we will get a complex-value hologram

$$
H_{\mathrm{cp}}=O R^{*}
$$

with which the reconstruction is free of the zero-order light and the twin image. Because the reconstruction is usually implemented by Fresnel diffraction, this kind of hologram is referred to as the complex Fresnel hologram. The complex hologram can be represented as a real amplitude function multiplying a pure phase function,

$$
H_{\mathrm{cp}}=\left|H_{\mathrm{cp}}\right| \times e^{i \Phi},
$$

where $\Phi$ is the phase difference between the object light and the reference light and $i$ denotes $\sqrt{-1}$. Thus, we can use two SLMs, one in AM mode and the other one in PM mode, to control the amplitude and the phase of the light, respectively. This is what has been done in [22-24], but cascading the two SLMs demands precise alignment. Alternatively, $H_{\mathrm{cp}}$ can be separated into two parts,

$$
H_{\mathrm{cp}}=\operatorname{Re}\left\{H_{\mathrm{cp}}\right\}+i \times \operatorname{Im}\left\{H_{\mathrm{cp}}\right\},
$$

where $\operatorname{Re}\{\cdot\}$ and $\operatorname{Im}\{\cdot\}$ stands for the real and the imaginary parts, respectively. It should be noticed that $\operatorname{Re}\left\{H_{\mathrm{cp}}\right\}$ and $\operatorname{Im}\left\{H_{\mathrm{cp}}\right\}$ are bipolar, i.e., the amplitude transmittance can be either positive or negative. The negative value means that the transmitting light suffers a $\pi$ phase shift. Therefore, $\operatorname{Re}\left\{H_{\mathrm{cp}}\right\}$ and $\operatorname{Im}\left\{H_{\text {cp }}\right\}$ cannot be faithfully displayed on AM SLMs. To avoid negative values, we define two amplitude holograms,

$$
H_{r}=\operatorname{Re}\left\{H_{\mathrm{cp}}\right\}+I_{0}, \quad H_{i}=\operatorname{Im}\left\{H_{\mathrm{cp}}\right\}+I_{0},
$$

where $I_{0}$ is a positive constant to bias the values of the holograms to be positive. In the previous method, one uses two SLMs both in the AM mode to respectively display the real and the imaginary parts of the complex hologram and uses a beam splitter to combine the two holograms so that a complex hologram can be synthesized [25-27]. In the Subsection 2.B, we show a new method to display a complex hologram using a single SLM.

\section{B. Synthetic Display System}

Figure 1 depicts the system for displaying a complex Fresnel hologram. We use a collimated laser light to illuminate the input pattern $t\left(x_{0}, y_{0}\right)$ displaying on the SLM, and the transmitting light is Fourier transformed by lens $L_{1}$. The complex amplitude at the Fourier plane is given by $[\underline{28}, \underline{29}]$

$$
\begin{array}{r}
u\left(x_{1}, y_{1}\right)=\frac{1}{i \lambda f_{1}} \mathcal{F}\left\{1 \times t\left(x_{0}, y_{0}\right)\right\} f_{f_{x}}=x_{1} / \lambda f_{1} \\
f_{y}=y_{1} / \lambda f_{1} \\
=\frac{1}{i \lambda f_{1}} T\left(f_{x}, f_{y}\right)=\frac{1}{i \lambda f_{1}} T\left(\frac{x_{1}}{\lambda f_{1}}, \frac{y_{1}}{\lambda f_{1}}\right)
\end{array}
$$

where $\mathcal{F}\{\cdot\}$ stands for Fourier transform operator, $\lambda$ is the wavelength of the laser, and $T\left(f_{x}, f_{y}\right)$ is the Fourier transform of $t\left(x_{0}, y_{0}\right)$ with $f_{x}$ and $f_{y}$ being the spatial frequencies along the $x$ and $y$ directions, respectively. At the Fourier plane, we interpose a sinusoidal amplitude grating. The complex amplitude at the output plane is then given by 


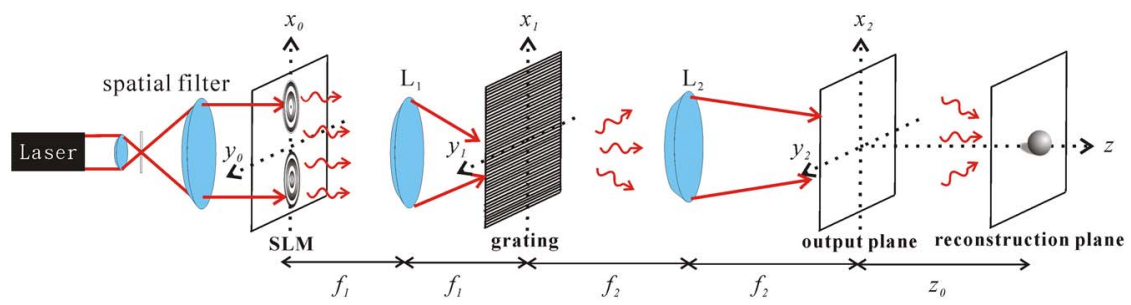

Fig. 1. (Color online) Schematic setup of the proposed display system for displaying a complex hologram.

$$
\begin{aligned}
u_{2}\left(x_{2}, y_{2}\right)= & \frac{1}{i \lambda f_{2}} \mathcal{F}\left\{\frac{1}{i \lambda f_{1}} T\left(\frac{x_{1}}{\lambda f_{1}}, \frac{y_{1}}{\lambda f_{1}}\right)\right. \\
& \left.\times\left[\frac{1}{2}+\frac{m}{2} \cos \left(2 \pi \frac{x_{1}}{\Lambda}\right)\right]\right\} f_{f_{x}}=x_{2} / \lambda f_{2},
\end{aligned}
$$

where $\Lambda$ is the period of the grating, and $m(0<m \leq$ 1 ) is the modulation depth of the grating. After some mathematical manipulations, Eq. (7) can be represented as

$$
\begin{aligned}
u_{2}\left(x_{2}, y_{2}\right)= & \frac{1}{M}\left\{\frac{1}{2} t\left(\frac{x_{2}}{M}, \frac{y_{2}}{M}\right)+\frac{m}{4} t\left(\frac{x_{2}}{M}+\lambda \frac{f_{1}}{\Lambda}, \frac{y_{2}}{M}\right)\right. \\
& \left.+\frac{m}{4} t\left(\frac{x_{2}}{M}-\lambda \frac{f_{1}}{\Lambda}, \frac{y_{2}}{M}\right)\right\},
\end{aligned}
$$

where $M=-f_{2} / f_{1}$ is the magnification of the system. We observe that three duplicates of the input pattern appear at the output plane. In Subsection 2.C, we design an input pattern with which the complex hologram can be synthesized at the output plane. Subsequently, the desired reconstructed real image can be observed behind the output plane.

\section{Two Amplitude Holograms as the Input Pattern to Form a Complex Hologram with a Bias Constant}

To realize the display of a complex hologram, the input pattern is designed as

$$
t\left(x_{0}, y_{0}\right)=H_{r}\left(x_{0}-d, y_{0}\right)+H_{i}\left(x_{0}+d, y_{0}\right) \times e^{i \pi / 2},
$$

where the $\pi / 2$ phase can be introduced with a tilted optical flat in front of $H_{i}$. By substituting Eq. (9) into Eq. (8), we obtain the optical field at the output plane as

$$
\begin{aligned}
u_{2}\left(x_{2}, y_{2}\right)= & \frac{1}{M}\left\{\frac{1}{2}\left[H_{r}\left(\frac{x_{2}}{M}-d, \frac{y_{2}}{M}\right)+i \times H_{i}\left(\frac{x_{2}}{M}+d, \frac{y_{2}}{M}\right)\right]\right. \\
& +\frac{m}{4}\left[H_{r}\left(\frac{x_{2}}{M}-d+\lambda \frac{f_{1}}{\Lambda}, \frac{y_{2}}{M}\right)\right. \\
& \left.+i \times H_{i}\left(\frac{x_{2}}{M}+d+\lambda \frac{f_{1}}{\Lambda}, \frac{y_{2}}{M}\right)\right] \\
& +\frac{m}{4}\left[H_{r}\left(\frac{x_{2}}{M}-d-\lambda \frac{f_{1}}{\Lambda}, \frac{y_{2}}{M}\right)\right. \\
& \left.\left.+i \times H_{i}\left(\frac{x_{2}}{M}+d-\lambda \frac{f_{1}}{\Lambda}, \frac{y_{2}}{M}\right)\right]\right\} .
\end{aligned}
$$

If the condition $d=\lambda f_{1} / \Lambda$ is held, Eq. (10) can be rewritten as

$$
\begin{aligned}
u_{2}\left(x_{2}, y_{2}\right)= & \frac{1}{M}\left\{\frac{m}{4}\left[H_{r}\left(\frac{x_{2}}{M}, \frac{y_{2}}{M}\right)+i \times H_{i}\left(\frac{x_{2}}{M}, \frac{y_{2}}{M}\right)\right]\right. \\
& +\frac{1}{2} H_{r}\left(\frac{x_{2}}{M}-d, \frac{y_{2}}{M}\right)+\frac{i}{2} \times H_{i}\left(\frac{x_{2}}{M}+d, \frac{y_{2}}{M}\right) \\
& +\frac{m}{4} H_{r}\left(\frac{x_{2}}{M}-2 d, \frac{y_{2}}{M}\right) \\
& \left.+\frac{i m}{4} \times H_{i}\left(\frac{x_{2}}{M}+2 d, \frac{y_{2}}{M}\right)\right\},
\end{aligned}
$$

where the first term is proportional to the magnified complex hologram plus a constant value, i.e., $H_{\mathrm{cp}}\left(\frac{x_{2}}{M}, \frac{y_{2}}{M}\right)+I_{0}(1+i)$, by recognizing the definition in Eq. (5), and other terms are higher diffraction orders. Thus, the window size $w$ of $H_{r}$ and $H_{i}$ should be smaller than $2 d(w<2 d)$ to avoid the interference between adjacent diffraction orders, and the desired optical field can be filtered through an aperture stop with width $|M| w$ at the center of the output plane. Finally, the constant value $I_{0}(1+i)$ can be easily removed by superimposing a plane wave on the synthesized complex hologram by a beam splitter because there is no alignment problem on it.

\section{Multiple Amplitude Holograms as the Input Pattern to Form a Pure Complex Hologram}

In Subsection 2.C, we use two holograms to synthesize a complex hologram. The principle can be easily understood from Eqs. (10) and (11). The sinusoidal grating at the Fourier plane produces three duplicates of the input patterns at the output plane. The first-order duplicate of $H_{r}$ and the minus first-order duplicate of $H_{i}$ overlap at the center of the output plane while we carefully shift the two holograms $H_{r}$ and $H_{i}$. Based on this idea, we can also combine multiple patterns by involving more diffraction orders of light. For example, we design an input pattern as

$$
\begin{aligned}
t\left(x_{0}, y_{0}\right)= & H_{r}\left(x_{0}-d, y_{0}\right)+H_{i}\left(x_{0}+d, y_{0}\right) \times e^{i \pi / 2} \\
& +H_{d c}\left(x_{0}, y_{0}\right) \times e^{i 5 \pi / 4},
\end{aligned}
$$

where $H_{\mathrm{dc}}=m I_{0} / \sqrt{2}$ is a designed neutral density aperture at the center of the input plane. Meanwhile, the window size of the three amplitude holograms should be smaller than $d(w<d)$ in order to avoid interference between adjacent diffraction orders. Consequently, at the output plane, the first diffraction order of $H_{r}\left(x_{0}-d, y_{0}\right)$, the minus first order of $H_{i}\left(x_{0}+d, y_{0}\right)$, and the zero order of $H_{\mathrm{dc}}\left(x_{0}, y_{0}\right)$ overlap 
at the center of the output plane to obtain a pure synthesized complex hologram given by

$$
\begin{aligned}
\frac{1}{M} & \left\{\frac{m}{4} H_{r}\left(\frac{x_{2}}{M}, \frac{y_{2}}{M}\right)+i \frac{m}{4} H_{i}\left(\frac{x_{2}}{M}, \frac{y_{2}}{M}\right)-\frac{1}{2}\right. \\
& \left.\times\left(\frac{1}{\sqrt{2}}+\frac{i}{\sqrt{2}}\right) H_{\mathrm{dc}}\left(\frac{x_{2}}{M}, \frac{y_{2}}{M}\right)\right\} \\
& =\frac{m}{4 M}\left[H_{r}\left(\frac{x_{2}}{M}, \frac{y_{2}}{M}\right)+i H_{i}\left(\frac{x_{2}}{M}, \frac{y_{2}}{M}\right)-(1+i) I_{0}\right] \\
& =\frac{m}{4 M} H_{\mathrm{cp}}\left(\frac{x_{2}}{M}, \frac{y_{2}}{M}\right),
\end{aligned}
$$

which is free of not only the twin image but also the zero-order light. In the above analysis, a sinusoidal amplitude grating is applied so that only three orders of the diffracted light as well as three patterns can be involved. If a sinusoidal phase grating or a Ronchi grating is applied, there will be more than three orders of diffracted light [29]. Thus, more than three patterns can be included in the system. The degree of freedom can be extended by involving more patterns. Nevertheless, the design of the input pattern becomes complicated because the diffraction efficiency varies at different diffraction orders. Besides, the total light efficiency of the system decreases because only a single order of the diffracted light is selected for each amplitude hologram to synthesize a complex hologram.

\section{Simulations}

\section{A. Two and Three Amplitude Holograms as the Input Pattern}

In this section, we show the simulations using two and three amplitude holograms as the input pattern. Our simulation parameters are described as follows. The wavelength is $0.633 \mu \mathrm{m}$ and the pixel size is $12.66 \mu \mathrm{m}$. The size of a single amplitude hologram is $500 \times 500$ pixels, and thus the window size is 500 pixels $(w=6.33 \mathrm{~mm})$. The shift of the holograms at the SLM is also 500 pixels $(d=6.33 \mathrm{~mm})$. The size of the SLM is $2000 \times 2000$ pixels and hence either two or three amplitude holograms can be displayed on it. We set $f_{1}=f_{2}=10 \mathrm{~cm}$ so that $M=-1$. Finally, the grating period is calculated to be $\Lambda=\lambda f_{1} / d=10 \mu \mathrm{m}$.

Figures 2(a)-2(c) show the object pattern, the amplitude hologram $H_{r}$, and the corresponding reconstructed image, respectively. The hologram, $H_{r}$, is obtained by adding a bias level $I_{0}$ to the real part of the diffraction field propagated from the object pattern, i.e.,

$$
H_{r}=\operatorname{Re}\{O\}+I_{0}=\operatorname{Re}\{u \otimes h\}+I_{0},
$$

where $u$ is the light field just behind the object pattern, $h$ is the spatial impulse response in Fourier optics [28,29], and " $\otimes$ " stands for the convolution operator $[28,29]$. So the hologram in Eq. (14) is an on-axis Fresnel hologram (taking $R^{*}=1$ ). The diffraction distance is $8 \mathrm{~cm}$, and thus the reconstruction distance $z_{0}$ is also $8 \mathrm{~cm}$. With reference to Fig. 2(c), the desired reconstructed image is blurred with the twin image and the zero-order light when a single amplitude hologram is reconstructed. We can use similar way to obtain $H_{i}$, and finally produce a complex pattern consisting of two holograms, shown in Fig. 3(a), on the input plane of the image processing system, where the two holograms have been displayed according to the design of Eq. (9). At the output plane, there are multiple diffraction orders, and we choose the central order of light, which is marked with the square in Fig. 3(b), for reconstruction. The reconstructed image is shown in Fig. 3(c). The object pattern is clearly retrieved without the twin image noise. However, the contrast (the intensity ratio of the reconstructed image and the background) is only 1.93 because the uniform bias persists. The bias light can be removed by involving a third uniform hologram, as described in Subsection 2.D [see Eq. (12)]. Figures 4(a) and 4(b) show the devised pattern, including three amplitude holograms and the synthesized complex hologram at the output plane, respectively. The bias light is successfully removed from the central order of light, and thus the contrast of the reconstructed image shown in Fig. 4(c) is ideally infinity.

\section{B. Two Binary Holograms as the Input Pattern}

In the previous discussions and simulations, the amplitude holograms are assumed to be gray tone. But, in Section 4 , we use two binary holograms for experimental demonstration because binary holograms can be produced swiftly with printers. In addition, with binary holograms, we could enhance the storage capacity of digital holograms and facilitate more

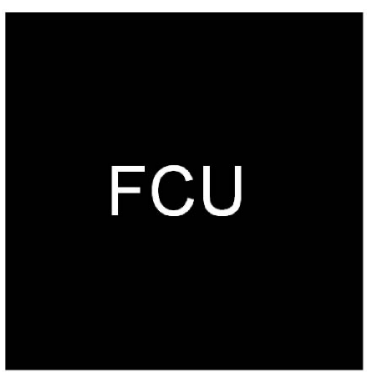

(a)

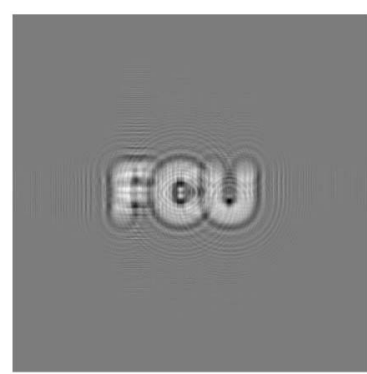

(b)

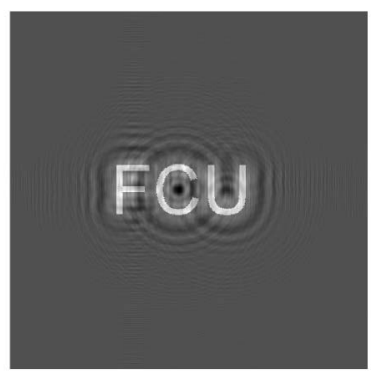

(c)

Fig. 2. (a) Original object pattern, (b) the amplitude hologram $H_{r}$, (c) intensity of the reconstructed optical field. 
efficient transmission of holograms [30]. To accomplish the binarization process, we first add a random phase to the object pattern, and then calculate the corresponding diffraction field. The binary holograms are obtained by binarizing the real and the imaginary parts of $H_{\mathrm{cp}}$ by the sign binarization method [30], i.e.,

$$
\begin{aligned}
& B_{r}=B^{0}\left\{\operatorname{Re}\left\{\left(u \cdot e^{i \theta}\right) \otimes h\right\}\right\}, \\
& B_{i}=B^{0}\left\{\operatorname{Im}\left\{\left(u \cdot e^{i \theta}\right) \otimes h\right\}\right\},
\end{aligned}
$$

where $\theta$ is a random function between 0 and $2 \pi$ and $B^{0}\{\cdot\}$ is the binarization operator with the threshold value being set to zero, i.e., if the input value is larger than zero, then the output value is 1 ; otherwise the output value is zero. The random phase can help expand the spectrum of the object light and reduces the edge effect caused by binarization [30]. The two binary holograms $B_{r}$ and $B_{i}$ are shown in Figs. 5(a) and $5(\mathrm{~b})$, respectively. Figure 5(c) is the reconstruction of a single binary hologram $B_{r}$, while Fig. $5(\mathrm{~d})$ shows the reconstruction of the synthesized complex hologram. Although some artifacts due to the binarization process exist, most of the background noise, such as the noise due to the twin image, has been removed.

\section{System Tolerance and Experimental Demonstration}

The quality of the reconstructed image decreases as the proposed system is not ideally aligned. First, the separation between the two amplitude holograms should be well controlled so that they can overlap at

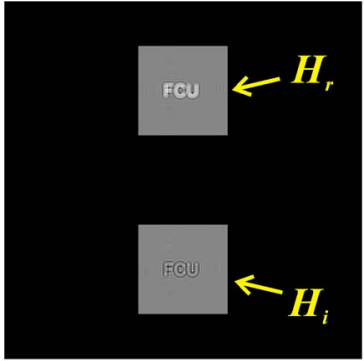

(a)

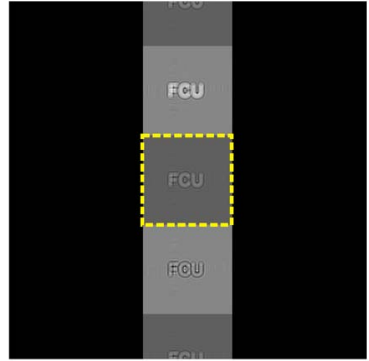

(b)

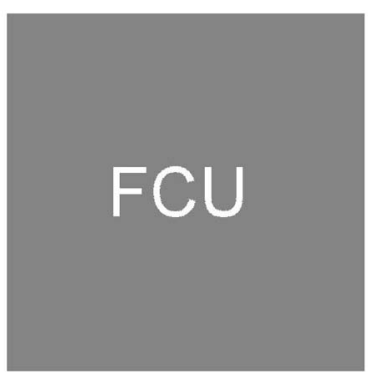

(c)

Fig. 3. (Color online) Modulus of the (a) designed input pattern at SLM and the (b) optical field at the output plane. The central portion of the light, which is marked with a yellow square, is the synthesized complex hologram. (c) Intensity of the reconstructed image at the reconstruction plane.

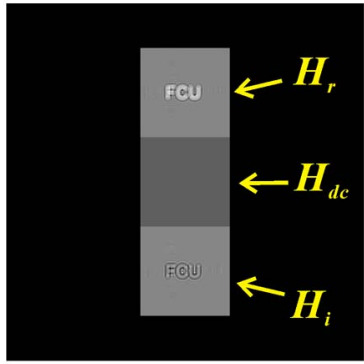

(a)

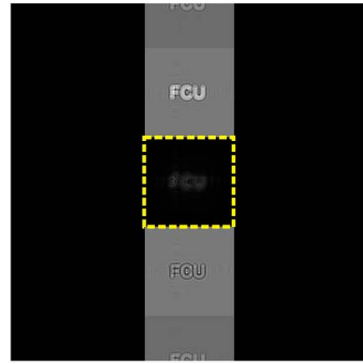

(b)

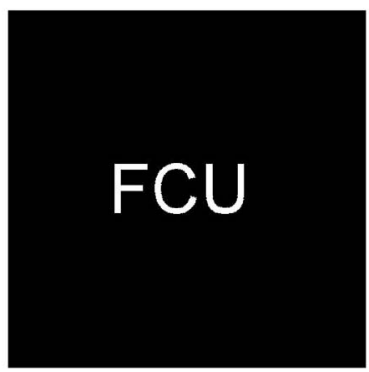

(c)

Fig. 4. (Color online) Same as Fig. 3, but in (a) the designed input pattern at SLM contains three amplitude holograms.

the output plane. Apparently, the separation error should be much smaller than 1 pixel to avoid the mismatch error. However, it should be noticed that the optical complex summation is at the $\left(x_{2}, y_{2}\right)$ plane, but the separation distance is adjusted at the $\left(x_{0}, y_{0}\right)$ plane. Besides, there can be a magnification between the two planes. Thus, it is possible to have a smaller pixel size at the $\left(x_{2}, y_{2}\right)$ plane while the pixel size of the SLM at the $\left(x_{0}, y_{0}\right)$ plane is larger for easy

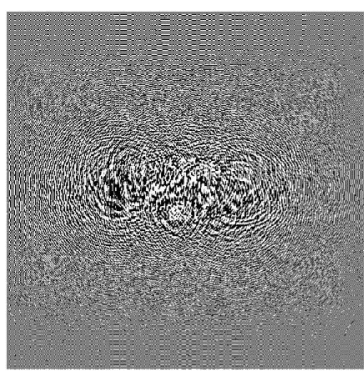

(a)

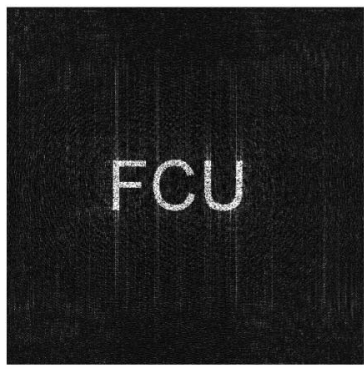

(c)

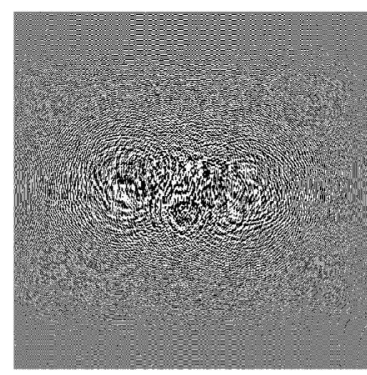

(b)

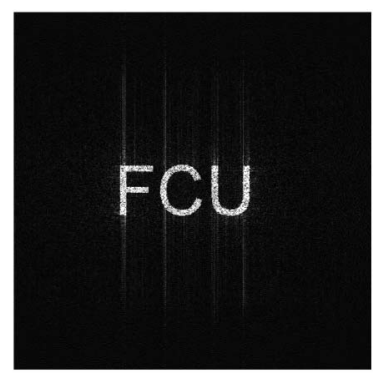

(d)
Fig. 5. (a) Binary hologram $B_{r}$, (b) binary hologram $B_{i}$, (c) image reconstructed using a single hologram $B_{r}$, (d) image reconstructed using two binary holograms and the proposed system. 


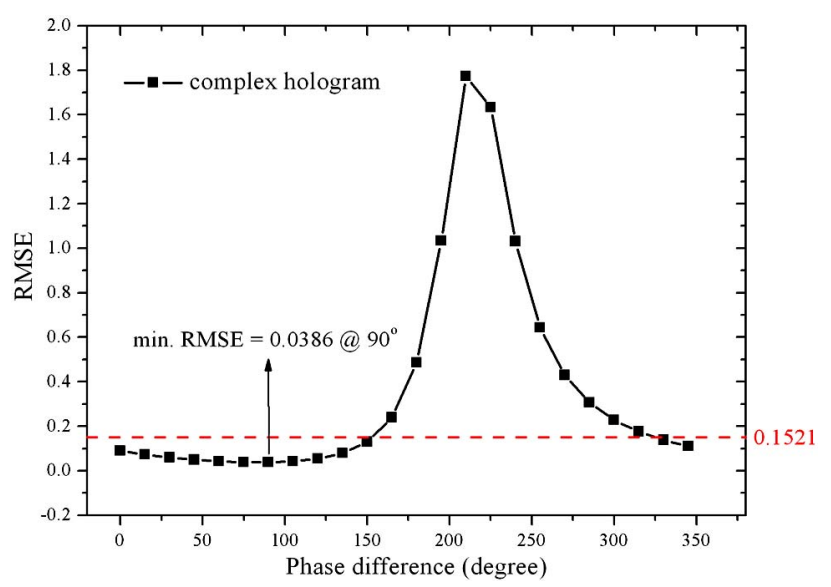

Fig. 6. (Color online) RMSE as a function of the reconstruction phase difference between two gray-tone holograms. The horizontal dashed line indicates the RMSE for a single gray-tone hologram.

alignment. The absolute transverse location of the amplitude holograms is not significant. It results in only a transverse shift of the synthesized complex hologram, but no mismatch error.

If the orientation of the grating at the Fourier plane is not along the $x_{1}$ axis (Fig. 1), the orientation error also results in a relative shift between the two images at the $\left(x_{2}, y_{2}\right)$ plane. In the small-angle approximation, the relative shift is only along the $y_{2}$ axis, and its magnitude is about $\frac{2 \lambda f_{2} \phi}{\Lambda}$, where $\phi$ is the angle between the grating vector and the $y_{1}$ axis. Again, the quality of the reconstructed image declines when the mismatch is larger than 1 pixel.

In practical optical system, the phase difference between the two amplitude holograms may deviate from $90^{\circ}$. The phase error also introduces error in the reconstructed image. Figure 6 shows the simula-

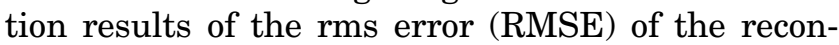
structed image when comparing with the original image as a function of the phase difference between two holograms in the reconstruction. In Fig. $\underline{6}$, we have used two bipolar gray-tone holograms to form

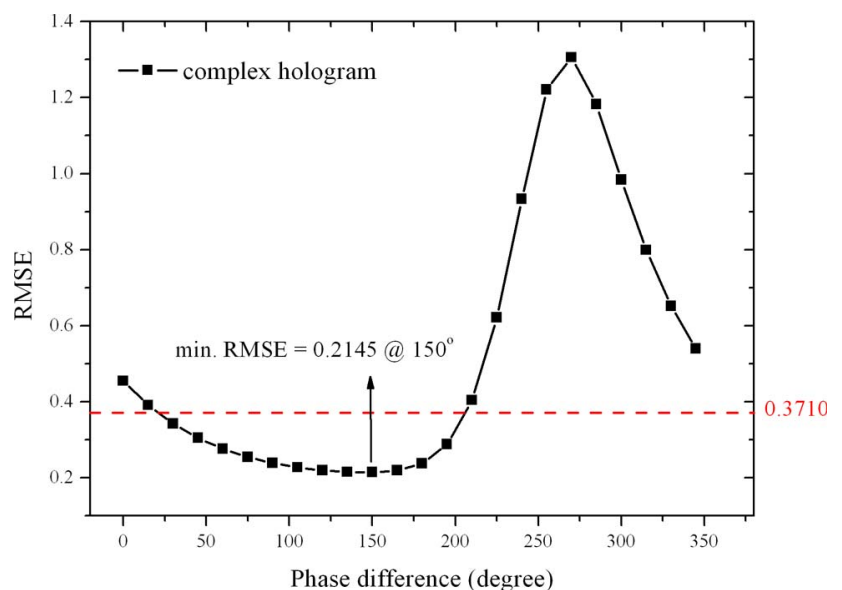

Fig. 7. (Color online) RMSE as a function of the reconstruction phase difference between two binary holograms. The horizontal dashed line indicates the RMSE for a single binary hologram. a complex hologram so that the zero-order light is directly removed. The minimum RMSE (best reconstruction quality) is achieved when the phase difference in the reconstruction is $90^{\circ}$. And, the minimum RMSE for the synthesized hologram is only one fourth of the RMSE for a single gray-tone hologram (marked with the red dashed line in Fig. 6). In Fig. 7, we have also plotted the RMSE of the reconstructed image as a function of the phase difference between two binary holograms. It is shown that the minimum RMSE is achieved while the phase difference is $150^{\circ}$, not $90^{\circ}(\mathrm{RMSE}=0.2389)$. It is argued that the error due to binarization can be partially cancelled by deliberately introducing a phase-difference error. However, the ability of such error reduction by using binary holograms is worse than that by using a single gray-tone hologram. Finally, in both cases (Figs. $\underline{6}$ and 7), the RMSE for a complex hologram is lower than that for a single amplitude hologram in a wide range of phase differences. Therefore, an accurate phase control is not critical in our proposed system.

In the experimental demonstration, we used an $\mathrm{He}-\mathrm{Ne}$ laser operated at $\lambda=0.633 \mu \mathrm{m}$ as the source; the focal lengths of the two lenses are respectively $f_{1}=10 \mathrm{~cm}$ and $f_{2}=10 \mathrm{~cm}$, and thus the magnification is $M=-1$. We interpose a Ronchi gating with period $\Lambda=10 \mu \mathrm{m}$ at the Fourier plane. Therefore, the transverse shift of a single hologram is $d=6.33 \mathrm{~mm}$, and the window size at the input plane should be smaller than $12.66 \mathrm{~mm}$. We have devised an input pattern consisting of two binary amplitude holograms, and printed it on a holographic plate (Slavich: VRP). The pixel pitch of the hologram is $2.67 \mu \mathrm{m}$, and the size of the two binary hologram is $1200 \times 1200$ pixels so that the window size is about $3.20 \mathrm{~mm}$. Because the magnification of the image processing system is -1 , the pixel size and the size of the aperture stop at the output plane are the same as those at the input plane. The photographs of the reconstructed images for a single binary hologram $B_{r}$ and the synthesized complex hologram are respectively shown in Figs. 8(a) and 8(b). We have captured the photographs by directly acquiring the diffraction pattern at the reconstruction plane with a lensless camera (Nikon D3100) at a fixed exposure time. In Fig. $8(b)$, we see that the reconstructed image is

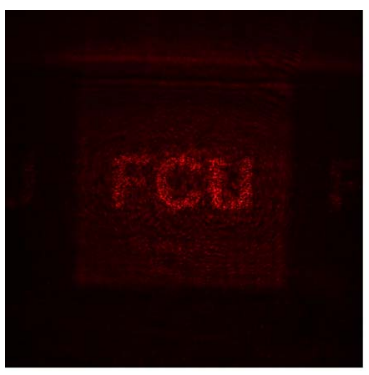

(a)

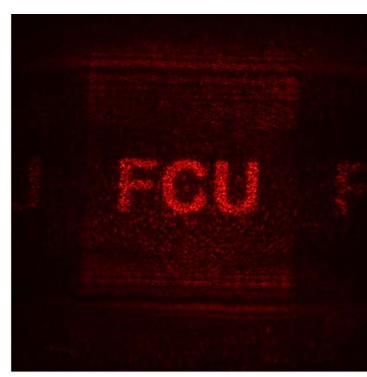

(b)
Fig. 8. (Color online) Photographs of the reconstructed images for (a) hologram $B_{r}$ and the (b) synthesized complex hologram. The original object pattern is the same as Fig. 2(a). 
enhanced and the background noise is partially suppressed and the contrast is improved.

\section{Conclusion}

We have proposed to use a single SLM to display a complex hologram that is free of the twin image and the zero-order light. The display architecture is a coherent image processing system in conjunction with a sinusoidal grating at the Fourier plane. The input pattern displayed on the SLM contains two or more transversely shifted amplitude holograms. The complex hologram can be synthesized at the output plane of the image processing system when the condition $d=\lambda f_{1} / \Lambda$, as discussed in Subsection 2.C, is held where $2 d$ is the separation between the two amplitude holograms. If the two amplitude holograms are used for synthesizing a complex hologram, the window size $w$ of each hologram should satisfy the condition $w<2 d$. If more than two amplitude holograms are used, the window size should satisfy $w<d$. The limitation of the window size reduces the field of view, but does not reduce the bandwidth of the holograms. Thus, the full bandwidth of the SLM can be utilized for displaying the two holograms. We have also analyzed the system tolerance of the proposed system. We found the quality of the reconstructed image is not really sensitive to the error of the phase difference between the two amplitude holograms. On the other hand, serious error always occurs while the relative shift between the two amplitude holograms is larger than 1 pixel. Consequently, a larger $\Lambda$ and a smaller $f_{2}$ can be selected to avoid the difficulty in alignment.

Although the proposed system is for displaying a complex hologram, it can be applied to combine two arbitrary holograms or patterns. For example, we can use the system to combine one hologram of the background and the other hologram of the foreground. Then the foreground and the background can be directly reconstructed in the same scene without additional calculation. We can also use the proposed system to increase the quantization levels of the displayed image. For example, any 8 bit image can be separated into two 7 bit images, and be displayed in a single 7 bit SLM. Then the original 8 bit image can be synthesized in our system. These possible applications are interesting and worth further investigation.

This work is supported by National Science Council of Taiwan (NSCT) under contact NSC 99-2221-E035-065. The authors thank C.-C. Lee for preparing the figures.

\section{References}

1. R. J. Collier, C. B. Burckhardt, and L. H. Lin, Optical Holography (Academic, 1971).

2. E. N. Leith and J. Upatnieks, "Reconstructed wavefronts and communication theory," J. Opt. Soc. Am. 52, 1123-1130 (1962).

3. Y. Takaki and N. Okada, "Hologram generation by horizontal scanning of a high-speed spatial light modulator," Appl. Opt. 48, 3255-3260 (2009).
4. Y. Takaki, M. Yokouchi, and N. Okada, "Improvement of grayscale representation of the horizontally scanning holographic display," Opt. Express 18, 24926-24936 (2010).

5. R. B. A. Tanjung, X. Xu, X. Liang, S. Solanki, Y. Pan, F. Farbiz, B. $\mathrm{Xu}$, and T.-C. Chong, "Digital holographic threedimensional display of 50-Mpixel holograms using a two-axis scanning mirror device," Opt. Eng. 49, 025801 (2010).

6. J. Hahn, H. Kim, Y. Lim, G. Park, and B. Lee, "Wide viewing angle dynamic holographic stereogram with a curved array of spatial light modulators," Opt. Express 16, 12372-12386 (2008).

7. K. Choi, J. Kim, Y. Lim, and B. Lee, "Full parallax viewingangle enhanced computer-generated holographic 3D display system using integral lens array," Opt. Express 13, 10494-10502 (2005).

8. T. Mishina, F. Okano, and I. Yuyama, "Time-alternating method based on single-sideband holography with half-zone-plate processing for the enlargement of viewing zones," Appl. Opt. 38, 3703-3713 (1999).

9. Y. Takaki and Y. Tanemoto, "Band-limited zone plates for single-sideband holography," Appl. Opt. 48, H64-H70 (2009)

10. T.-C. Poon, T. Kim, G. Indebetouw, B. W. Schilling, M. H. Wu, K. Shinoda, and Y. Suzuki, "Twin-image elimination experiments for three-dimensional images in optical scanning holography," Opt. Lett. 25, 215-217 (2000).

11. B. E. A. Saleh and K. Lu, "Theory and design of the liquid crystal TV as an optical spatial phase modulator," Opt. Eng. 29, 240-246 (1990).

12. J. A. Coy, M. Zaldarriaga, D. F. Grosz, and O. E. Martinez, "Characterization of a liquid crystal television as a programmable spatial light modulator," Opt. Eng. 35, 15-19 (1996).

13. P. Birch, R. Young, C. Chatwin, M. Farsari, D. Budgett, and J. Richardson, "Fully complex optical modulation with an analogue ferroelectric liquid crystal spatial light modulator," Opt. Commun. 175, 347-352 (2000).

14. V. Arrizón, G. Méndez, and D. Sánchez-de-La-Llave, "Accurate encoding of arbitrary complex fields with amplitude-only liquid crystal spatial light modulators," Opt. Express 13, 7913-7927 (2005).

15. R. W. Cohn and M. Liang, "Pseudorandom phase-only encoding of real-time spatial light modulators," Appl. Opt. 35, 24882498 (1996).

16. V. Arrizón, "Complex modulation with a twisted-nematic liquid-crystal spatial light modulator: double-pixel approach," Opt. Lett. 28, 1359-1361 (2003).

17. V. Arrizón, "Optimum on-axis computer-generated hologram encoded into low-resolution phase-modulation devices," Opt. Lett. 28, 2521-2523 (2003).

18. V. Arrizón, L. A. González, R. Ponce, and A. Serrano-Heredia, "Computer-generated holograms with optimum bandwidths obtained with twisted-nematic liquid-crystal displays," Appl. Opt. 44, 1625-1634 (2005).

19. E. Buckley, A. Cable, N. Lawrence, and T. Wilkinson, "Viewing angle enhancement for two-and three-dimensional holographic displays with random superresolution phase masks," Appl. Opt. 45, 7334-7341 (2006).

20. D. Mendlovic, G. Shabtay, U. Levi, Z. Zalevsky, and E. Marom, "Encoding technique for design of zero-order (on-axis) Fraunhofer computer-generated holograms," Appl. Opt. 36, 8427-8434 (1997).

21. J. A. Davis, D. M. Cottrell, J. Campos, M. J. Yzuel, and I. Moreno, "Encoding amplitude information onto phase-only filters," Appl. Opt. 38, 5004-5013 (1999).

22. L. G. Neto, D. Roberge, and Y. Sheng, "Full-range, continuous, complex modulation by the use of two coupled-mode liquidcrystal televisions," Appl. Opt. 35, 4567-4576 (1996).

23. R. Tudela, I. Labastida, E. Martín-Badosa, S. Vallmitjana, I. Juvells, and A. Carnicer, "A simple method for displaying 
Fresnel holograms on liquid crystal panels," Opt. Commun. 214, 107-114 (2002).

24. M.-L. Hsieh, M.-L. Chen, and C.-J. Cheng, "Improvement of the complex modulated characteristic of cascaded liquid crystal spatial light modulators by using a novel amplitude compensated technique," Opt. Eng. 46, 070501 (2007).

25. R. Tudela, E. Martín-Badosa, I. Labastida, S. Vallmitjana, I. Juvells, and A. Carnicer, "Full complex Fresnel holograms displayed on liquid crystal devices," J. Opt. A 5, S189-S194 (2003).

26. T. Ra, M.-B. Estela, L. Ignasi, V. Santiago, and C. Artur, "Wavefront reconstruction by adding modulation capabilities of two liquid crystal devices," Opt. Eng. 43, 2650-2657 (2004).
27. S.-G. Kim, B. Lee, and E.-S. Kim, "Removal of bias and the conjugate image in incoherent on-axis triangular holography and real-time reconstruction of the complex hologram," Appl. Opt. 36, 4784-4791 (1997).

28. T.-C. Poon, Optical Scanning Holography with MATLAB (Springer, 2007).

29. J. W. Goodman, Introduction to Fourier Optics (McGrawHill, 2005).

30. P. Tsang, T.-C. Poon, W. K. Cheung, and J.-P. Liu, "Computer generation of binary Fresnel holography," Appl. Opt. 50, B88-B95 (2011). 